PROCEEDINGS OF THE

AMERICAN MATHEMATICAL SOCIETY

Volume 140, Number 4, April 2012, Pages 1309-1320

S 0002-9939(2011)10985-7

Article electronically published on July 29,2011

\title{
EVERY EXPANDING MEASURE HAS THE NONUNIFORM SPECIFICATION PROPERTY
}

\author{
KRERLEY OLIVEIRA
}

(Communicated by Bryna Kra)

\begin{abstract}
Exploring abundance and nonlacunarity of hyperbolic times for endomorphisms preserving an ergodic probability with positive Lyapunov exponents, we obtain that there are periodic points of period growing sublinearly with respect to the length of almost every dynamical ball. In particular, we conclude that any ergodic measure with positive Lyapunov exponents satisfies the nonuniform specification property. As consequences, we (re)obtain estimates on the recurrence to a ball in terms of the Lyapunov exponents, and we prove that any expanding measure is the limit of Dirac measures on periodic points.
\end{abstract}

\section{INTRODUCTION}

A basic problem in dynamical systems and its applications is to study the existence and abundance of periodic points and understand its distribution on the underlying space. In simple terms, for a differentiable map $f: M \rightarrow M$ in a Riemann manifold $M$, one wants to analyse which conditions on $f$ and its derivatives ensure the existence of periodic points and how they are distributed in $M$.

For uniformly hyperbolic maps, Bowen established in [5] that the asymptotical exponential growth of the set $P_{n}(f)$ of periodic points of period $n$ was determined by the topological entropy

$$
\lim _{n \rightarrow \infty} \frac{\log P_{n}(f)}{n}=h_{\text {top }}(f) .
$$

He introduced the important notion of specification by periodic orbits and proved a number of important results concerning the uniqueness and the ergodic properties of equilibrium states, asymptotic growth and the limit distribution of periodic orbits and so on, for Axiom A diffeomorphisms and flows ([5]). In words, a system has the specification property if a (small) error is fixed; given a piece of orbit of size $n$, there exists a periodic point that follows (up to this error) this orbit up to the moment $n$ and has period $n+K$, where $K$ depends only on the error and does not depend on $n$.

Received by the editors July 28, 2010 and, in revised form, December 22, 2010 and December 27, 2010.

2010 Mathematics Subject Classification. Primary 37D25, 37A99.

Key words and phrases. Periodic points, Lyapunov exponents.

This work was partially supported by CNPq, CAPES, FAPEAL, INCTMAT and PRONEX.

(C)2011 American Mathematical Society Reverts to public domain 28 years from publication 
More precisely, we say that $f$ has the specification property if there exists $\epsilon_{0}>0$ such that for all $x \in M$ and $0<\epsilon<\epsilon_{0}, n \geq 1$, there exists a periodic point $p \in M$ such that

- $d\left(f^{i}(p), f^{i}(x)\right)<\epsilon$, for $i=0, \ldots, n$;

- $p$ has period less than $n+K$, with $K=K(\epsilon)$ depending only on $\epsilon$.

For convenience, we define the dynamical ball of size $\epsilon$ and length $n$ by

$$
B_{n}(x, \epsilon)=\bigcap_{k=0}^{n} f^{-k}\left(B\left(f^{k}(x), \epsilon\right)\right),
$$

and this can be rephrased just saying that there exists a periodic point of period less than $n+K(\epsilon)$ in $B_{n}(x, \epsilon)$.

Beyond the uniformly hyperbolic setting, the understanding of periodic orbits and their structure is much less developed. A remarkable work stablishing connections between Lyapunov exponents of a given measure and periodic points and their distribution was obtained by Katok in 8 . There, he proved a technical lemma known as the Katok Closing Lemma: roughly, it tells us that if $f$ is a $C^{1+\alpha}$ diffeomorphism and $x$ is a recurrent point in a Pesin's block such that $f^{n}(x)$ is in the same Pesin's block, the orbit of $x$ up to the moment $n$ can be shadowed by a periodic point $p$ with period depending only on the shadowing constant and the Pesin block, not on $n$ (see 8], Section 3 for precise statements). Recent improvements of this result include the papers [15, 7].

Here, we improve part of this result relaxing the hypothesis in 8 and obtaining a quantitative version of Katok's Closing Lemma for nonuniformly expanding maps preserving an ergodic measure with positive exponents. This can be regarded as an extension of the Bowen specification property in a measure-theoretical setting.

Consider an ergodic invariant measure $\mu$ with only positive Lyapunov exponents for any $C^{1}$ endomorphisms $f$ with nonflat critical set. We are able to show that for $\mu$ almost every point, given a natural number $n$ and $\epsilon>0$, there exists a periodic point $p$ on the dynamical ball $B_{n}(x, \epsilon)$ with period $K(n, \epsilon)$ that grows asymptotically like $n$ at infinity.

This generalizes the Katok Closing Lemma in several ways. First, we are able to deal with $C^{1}$ maps, instead of $C^{1+\alpha}$ maps, since we do not need to make use of Pesin's theory. Moreover, we are able not only to prove the existence of a shadowing point but to obtain quantitative estimates on its period. As a consequence of our result, we are able to obtain estimates on Poincaré recurrence in terms of the Lyapunov exponents. Let us describe it in detail.

The studies of recurrence and return times are among the most prolific tools for a better understanding of the statistical properties of dynamical systems. Themost basic concept in this context is the Poincaré recurrence of a set. Given a measurable dynamical system $(M, \mu, f)$ and a measurable set $A \subset M$, we define its Poincaré recurrence as

$$
\tau(A):=\inf \left\{n \in \mathbb{N} ; f^{n}(A) \cap A \neq \emptyset\right\} .
$$

In the literature, many relations have been established between recurrence times and other important aspects of dynamical systems such as entropy, Hausdorff dimension, mixing properties and Lyapunov exponents.

In order to grasp a finer understanding of these relations, it is useful to consider return times associated to shrinking neighborhoods such as decreasing sequences of balls or cylinders. 
Closely related to the Poincaré recurrence of $B_{n}(x, \epsilon)$, we may ask for the existence of periodic points in this dynamical ball. Sometimes it is possible to find a periodic point $z$ in a given ball but often its period is unrelated to $n$ or $\epsilon$. In the scenario we are facing, we can restrict our attention to investigate how frequently dynamical balls contain periodic points of (at some extent) a controlled period. This gives place to a notion of specification that bounces back to Bowen itself [5, 6].

In $[4$ is presented the nonuniform counterpart of these notions allowing that the radius $\epsilon$ in equation (11) decreases with $n$. To begin with, let $q: M \rightarrow[0, \infty)$ be an $\eta$-slowly varying function, that is to say, a function satisfying $q(f(x)) \leq e^{\eta} q(x)$, for all $x \in M$ and some fixed $\eta>0$. A $(n, \epsilon, q)$ nonuniform dynamical ball is defined as

$$
\widetilde{B}_{n}(x, \epsilon)=\bigcap_{k=0}^{n} f^{-k}\left(B\left(f^{k}(x), \epsilon q\left(f^{k}(x)\right)^{-2}\right)\right) .
$$

We say that $(f, \mu)$ has the nonuniform specification property if for $\mu$-almost everywhere, given an $\eta$-slowing varying function $q$, the ball $\widetilde{B}_{n}(x, \epsilon)$ contains a periodic point whose period is less than $n+K(n, \epsilon, \eta)$ satisfying

$$
\lim _{\eta \rightarrow 0} \limsup _{n \rightarrow \infty} \frac{K(n, \epsilon, \eta)}{n}=0 \text {. }
$$

In the same work, Saussol et al. 4 proved that the nonuniform specification property implies an estimate of the recurrence time for arbitrary positive $\mu$-measure sets in terms of Lyapunov exponents.

Here we are able to obtain a more natural result showing that positiveness of all Lyapunov exponents implies the nonuniform specification property. The main idea that we use to obtain this result is the notion of hyperbolic time. This notion has been used by many authors to obtain statistical properties of dynamical systems, such as existence and uniqueness of SRB measures (2]), stochastic stability (1]), infinite Markov Partitions ([13) and equilibrium states ([9, 10). In this paper, we prove that almost every point with respect to any ergodic measure with positive exponents has a nonlacunary sequence of hyperbolic times. Let us state our main result:

Theorem A. Let $f: M \rightarrow M$ be a $C^{1}$ map with nonflat critical set $\mathcal{C}$. Any $f$-invariant expanding measure $\mu$ satisfies the nonuniform specification property.

See Section 2 for precise definitions. Using Theorem $\mathrm{A}$ above and the Theorem of [4, we obtain that:

Corollary 1.1. Let $f: M^{n} \rightarrow M^{n}$ be a $C^{1}$ map with nonflat critical set $\mathcal{C}$, preserving an $f$-invariant expanding measure $\mu$ with Lyapunov exponents $0<\lambda_{1} \leq$ $\cdots \leq \lambda_{n}$. For $\mu$ almost every $x \in M$,

$$
\limsup _{r \rightarrow 0} \frac{\tau(B(x, r))}{-\log r} \leq \frac{1}{\lambda_{1}} .
$$

Moreover, if we assume that $h_{\mu}(f)>0$, the following inequality also holds true:

$$
\frac{1}{\lambda_{n}} \leq \liminf _{r \rightarrow 0} \frac{\tau(B(x, r))}{-\log r} .
$$


It is interesting to observe that the inequalities presented in Corollary 1.1 may be attained, but there are examples where these inequalities are strict. In fact, there exists a linear expanding map on the two-dimensional torus preserving the Lebesgue measure and with Lyapunov exponents $0<\lambda_{1}<\lambda_{2}$ such that

$$
\frac{1}{\lambda_{2}}<\frac{2}{\lambda_{1}+\lambda_{2}}=\lim _{r \rightarrow 0} \frac{\tau(B(x, r))}{-\log r}<\frac{1}{\lambda_{1}}
$$

for Lebesgue almost every point $x \in M$. See Section 4 of [4], for further details and some other examples.

Another interesting consequence of Theorem $\mathrm{A}$ above is the fact that every expanding measure is approximated in the weak ${ }^{\star}$ topology by Dirac measures at periodic points.

Corollary 1.2. Let $f: M \rightarrow M$ be a $C^{1}$ map with nonflat critical set $\mathcal{C}$, preserving an $f$-invariant expanding measure $\mu$. Then, $\mu$ is the weak $k^{\star}$ limit of Dirac measures at periodic points.

We point out that in [16] the author make use of the notion of nonuniform specification to establish bounds for the measure of deviation sets associated to continuous observables with respect to not necessarily invariant weak Gibbs measures. Under some mild assumptions, he obtained upper and lower bounds for the measure of deviation sets of some nonuniformly expanding maps, including quadratic maps and robust multidimensional nonuniformly expanding local diffeomorphisms. To describe more precisely the notions in this introduction, we recall some basic notions in Ergodic Theory in the next section.

\section{Preliminaries}

The Multiplicative Ergodic Theorem, due to Oseledets, says that if $\mu$ is an ergodic measure such that $\log ^{+}\|D f(x)\|$ is integrable, then there exist numbers

$$
\bar{\lambda}_{1}>\cdots>\bar{\lambda}_{k}
$$

such that for $\mu$-almost every $x \in M$, there exists a flag

$$
\{0\}=E_{1}^{x} \subset E_{2}^{x} \subset \cdots \subset E_{k(x)}^{x}=T_{x} M
$$

depending measurably on the point and invariant by $D f$, such that for $i=1,2, \ldots$, $k-1$

$$
\lim _{n \rightarrow \infty} \frac{1}{n}\left\|D f^{n}(x) v\right\|=\bar{\lambda}_{i}, \quad \text { for all } \quad v \in E_{i+1}^{x} \backslash E_{i}^{x} .
$$

Note that the last expression implies that each $\bar{\lambda}_{i}$ is an $f$-invariant function, i.e., $\bar{\lambda}_{i}(x)=\bar{\lambda}_{i}(f(x))$. In particular, if $\mu$ is ergodic the functions $\bar{\lambda}_{i}$ are constant almost everywhere. Let

$$
\lambda_{1}(x) \leq \lambda_{2}(x) \leq \cdots \leq \lambda_{d}(x)
$$

be the numbers $\bar{\lambda}_{j}(x)$, in a nondecreasing order, and each repeated with multiplicity $\operatorname{dim} E_{i+1}^{x}-\operatorname{dim} E_{i}^{x}$. These numbers are called the Lyapunov exponents of $f$ at the point $x$ and $\sum_{i=1}^{d} \lambda_{i}=\int \log \|\operatorname{det} D f(x)\| d \mu$. See [11] or [3], p. 134, for more details.

Since $f$ is $C^{1}$ and $M$ is compact, $\|D f(x)\|$ is uniformly bounded from above. Thus, the integrability condition on the multiplicative ergodic theorem above $\log ^{+}\|D f(x)\|$ always holds true. 
Definition 2.1. A map $f: M \rightarrow M$ is strongly transitive on a set $X$ if for any open set $U \subset M$ such that $U \cap X \neq \emptyset$, there exists $n \in \mathbb{N}$ such that $X \subset \bigcup_{j=0}^{n} f^{j}(U)$.

We recall that the support of an invariant measure $\mu$ is the full measure set $\operatorname{supp}(\mu)$ of all points such that any neighbourhood has positive measure.

Definition 2.2. We say that an ergodic f-invariant probability is an expanding measure if $\log ^{+}\|D f(x)\|$ is integrable, all its Lyapunov exponents are positive and $f$ is strongly transitive on the support of $\mu$.

Let us now present a family of maps introduced in [2].

Definition 2.3. Given a $C^{1}$ map $f: M \rightarrow M$, we say that $\mathcal{C} \subset M$ is a $\beta$-nondegenerate critical set if there exists $B>0$ such that the following three conditions hold.

(1) $\frac{1}{B} \operatorname{dist}(x, \mathcal{C})^{\beta} \leq \frac{\|D f(x) v\|}{\|v\|} \leq B \operatorname{dist}(x, \mathcal{C})^{-\beta}$, for all $v \in T_{x} M$ and $x \in$ $M \backslash \mathrm{C}$.

For every $x, y \in M \backslash \mathcal{C}$ with $\operatorname{dist}(x, y)<\operatorname{dist}(x, \mathcal{C}) / 2$, we have:

(2) $\quad\left|\log \left\|D f(x)^{-1}\right\|-\log \left\|D f(y)^{-1}\right\|\right| \leq \frac{B}{\operatorname{dist}(x, \mathcal{C})^{\beta}} \operatorname{dist}(x, y)$.

(3) There exists $K>0$ such that $\|\operatorname{det} D f(x)\|<K \operatorname{dist}(x, \mathcal{C})^{\beta}$ for $x \in M \backslash \mathfrak{C}$.

A map $f: M \rightarrow M$ will be called nonflat if $f$ is a local $C^{1}$ diffeomorphism in the whole manifold except in a $\beta$-nondegenerate critical set $\mathcal{C} \subset M$, for some $\beta$.

\section{Positive exponents And hyperbolic times}

Throughout we assume that $f: M \rightarrow M$ is a $C^{1}$ map with nonflat critical set $\mathcal{C}$ which preserves an expanding ergodic invariant measure $\mu$. We also assume that $f$ is strongly transitive on the support of $\mu$. It is well known (see [12) that expanding measures admit invariant unstable local manifolds at almost every point. However, the dependence of these manifolds with the base point is just measurable and several of its features are not suitable for computations. In particular, the size of this local manifold is just a measurable function, and this is an additional challenge when we need to handle these objects. In the next definition, we introduce a concept that addresses some of these difficulties.

Definition 3.1. Given $c>0$ and $\delta>0$, we say that $n$ is a $(c, \delta)$-hyperbolic time for a point $x \in M$ if we can find a neighborhood $V_{n}(x)$ of $x$ such that $f^{n}: V_{n}(x) \rightarrow$ $B_{\delta}\left(f^{n}(x)\right)$ is a homeomorphism with the property that given $x_{1}, x_{2} \in V_{n}(x)$, then

$$
\operatorname{dist}\left(f^{k}\left(x_{1}\right), f^{k}\left(x_{2}\right)\right) \leq e^{-2 c(n-k)} \operatorname{dist}\left(f^{n}\left(x_{1}\right), f^{n}\left(x_{2}\right)\right),
$$

for all $0 \leq k \leq n$.

In this context, we call $V_{n}(x)=f^{-n}\left(B_{\delta}\left(f^{n}(x)\right)\right)$ the hyperbolic pre-ball of length $n$ and radius $\delta$ at $x$.

Definition 3.2. We consider $H_{n}(c, \delta, f)$ to be the set of points $x$ such that $n$ is a hyperbolic time of $x$ and define

$$
H(c, \delta, f):=\bigcap_{m \geq 1} \bigcup_{n \geq m} H_{n}(c, \delta, f)
$$

to be the set of points with infinitely many $(c, \delta)$-hyperbolic times for $f$. 
Definition 3.3. Given $0<\theta<1$, let $H^{\theta}(c, \delta, f)$ be the set of points with frequency of $(c, \delta)$-hyperbolic times at least $\theta$ :

$$
H^{\theta}(c, \delta, f):=\left\{x \in H(c, \delta, f): \limsup _{n \rightarrow \infty} \frac{\#\left\{k: 1 \leq k \leq n, x \in H_{k}(c, \delta, f)\right\}}{n} \geq \theta\right\} .
$$

From [2, we have a sufficient criterium for an abundance of $(c, \delta)$-hyperbolic times at a point $x$. We say that $f$ is asymptotically c-expanding at a point $x$ if

$$
\limsup _{n \rightarrow \infty} \frac{1}{n} \sum_{i=0}^{n-1} \log \left\|D f\left(f^{i}(x)\right)^{-1}\right\|^{-1}>4 c .
$$

Furthermore, we say that the point $x$ satisfies the condition of slow approximation to the critical set if for all $\epsilon>0$ there exists $\delta>0$ such that

$$
\limsup _{n \rightarrow \infty} \frac{1}{n} \sum_{i=0}^{n-1}-\log \operatorname{dist}_{\delta}\left(f^{i}(x), \mathcal{C}\right) \leq \epsilon .
$$

Here, $\operatorname{dist}_{\delta}$ is the $\delta$-truncated distance:

$$
\operatorname{dist}_{\delta}(x, \mathcal{C}):=\operatorname{dist}(x, \mathcal{C}), \quad \text { whenever } \operatorname{dist}(x, \mathcal{C})<\delta \text { and } 1 \text { otherwise. }
$$

Lemma 3.4. There exists $\delta>0$ and $\theta>0$ depending only on $c$ and $f$ such that if $x$ is a point satisfying equations (2) and (3), then $x$ belongs to $H^{\theta}(c, \delta, f)$.

Proof. The result follows from Pliss' Lemma [14]. For details see [2, Corollary 3.2].

The abundance of hyperbolic times is given by

Lemma 3.5. Given $\mu$ an ergodic expanding measure, there exists $\ell \in \mathbb{N}$, and real numbers $c, \theta, \delta>0$ such that

$$
\mu\left(H^{\theta}\left(c, \delta, f^{\ell}\right)\right)=1 .
$$

Proof. We observe that since the Lyapunov exponent of $\mu$ is positive, we may find a constant $c>0$ such that for almost every $x \in M$, there exists $k_{0}(x) \in \mathbb{N}$ such that if $n \geq k_{0}(x)$, then $\left\|D f^{n}(x) v\right\| \geq e^{8 c n}\|v\|$, for every $v \in T_{x} M$. This is equivalent to $\left\|D f^{n}(x)^{-1}\right\| \leq e^{-8 c n}$. Denote by $A_{k}$ the set

$$
A_{k}=\left\{x \in M ; n_{0}(x) \leq k\right\} .
$$

It is clear that $\mu\left(A_{k}^{c}\right)$ goes to zero when $k$ goes to infinity. Observe that

$$
\begin{aligned}
\int_{M} \frac{1}{k} \log \left\|D f^{k}(x)^{-1}\right\| d \mu & =\int_{A_{k}} \log \frac{1}{k}\left\|D f^{k}(x)^{-1}\right\| d \mu+\int_{A_{k}^{c}} \log \frac{1}{k}\left\|D f^{k}(x)^{-1}\right\| d \mu \\
& \leq-8 c \mu\left(A_{k}\right)+\int_{A_{k}^{c}} \frac{1}{k} \log \left\|D f^{k}(x)^{-1}\right\| d \mu .
\end{aligned}
$$

On the other hand,

$$
\int_{A_{k}^{c}} \frac{1}{k} \log \left\|D f^{k}(x)^{-1}\right\| d \mu \leq \int_{A_{k}^{c}} \frac{1}{k} \sum_{i=0}^{k-1} \log \left\|D f\left(f^{i}(x)\right)^{-1}\right\| d \mu .
$$


We observe that $\log \left\|D f(x)^{-1}\right\|$ is integrable. Indeed, using condition (1) of definition 2.3 we can promptly check that

$$
\frac{1}{B} \operatorname{dist}(x, \mathcal{C})^{\beta} \leq\left\|D f(x)^{-1}\right\| \leq B \operatorname{dist}(x, \mathcal{C})^{-\beta},
$$

for all $x \in M \backslash \mathcal{C}$. Now, to conclude that $\log \left\|D f(x)^{-1}\right\|$ is $\mu$-integrable, we just need to prove that $\log \operatorname{dist}(x, \mathcal{C})$ is $\mu$-integrable. In fact, from condition (3) of Definition 2.3 we have that $\log \|\operatorname{det} D f(x)\| \leq \log K+\beta \log \operatorname{dist}(x, \mathrm{C})$. Using the Multiplicative Ergodic Theorem, we have that

$$
\begin{aligned}
-\infty<\sum_{i=1}^{d} \lambda_{i}=\int \log \|\operatorname{det} D f(x)\| & \leq \log K+\beta \int \log \operatorname{dist}(x, \mathcal{C}) \\
& <\log K+\beta \log \operatorname{diam}(M),
\end{aligned}
$$

where $\lambda_{1} \leq \cdots \leq \lambda_{d}$ are the Lyapunov exponents of $\mu$.

Now, using that $\log \left\|D f(x)^{-1}\right\|$ is integrable, by Birkhoff's Ergodic Theorem, the function $h_{k}(x)=\frac{1}{k} \sum_{i=0}^{k-1} \log \left\|D f\left(f^{i}(x)\right)^{-1}\right\|$ converges in $L^{1}(d \mu)$ to some function $\varphi$. Using that $\mu\left(A_{k}^{c}\right)$ goes to zero when $k$ goes to infinity, we have that

$$
\lim _{k \rightarrow \infty} \int_{A_{k}^{c}} \frac{1}{k} \log \left\|D f^{k}(x)^{-1}\right\| d \mu=0 .
$$

Observing this and equation (44) above, there exists $l_{0}$ such that if $\ell \geq l_{0}$,

$$
\int_{M} \frac{1}{\ell} \log \left\|D f^{\ell}(x)^{-1}\right\| d \mu<-4 c<0
$$

In particular, using Birkhoff's Ergodic Theorem once more, condition (2) holds for the function $f^{\ell}$ at almost every point $x \in M$. Since $\mu$ is an ergodic measure and has finite Lyapunov exponents with respect to $\mu$, we have by Lemma 10.2 of [13] that condition (3) holds for $f^{\ell}$ at almost every point. Since conditions (2) and (3) are satisfied, we have by Lemma 3.4 that there exists $\theta>0$ such that almost every $x \in M$ belongs to $H^{\theta}\left(c, \delta, f^{\ell}\right)$.

We fix $\ell, c, \delta, \theta>0$ as in the lemma above, and consider $g=f^{\ell}$. When we say just hyperbolic time, we mean $(c, \delta)$-hyperbolic time with respect to $g$. Define the first hyperbolic time function $n_{1}: H^{\theta}(c, \delta, g) \rightarrow \mathbb{N}$ setting $n_{1}(x)$ as the first hyperbolic time of $x$.

Remark 3.6. This follows from the fact that if $m$ is a hyperbolic time for $x$ and $n$ is a hyperbolic time for $g^{m}(x)$, then $n+m$ is a hyperbolic time for $x$; if we denote by $n_{1}(x)<n_{2}(x)<\ldots$ the sequence of hyperbolic times of $x$, then

$$
n_{1}\left(g^{n_{i}(x)}(x)\right)=n_{i+1}(x)-n_{i}(x) .
$$

For the next result we make use of [13. Lemma 4.7].

Lemma 3.7 ([13, Lemma 4.7]). Let $\left(G_{j}\right)_{j \in \mathbb{N}}$ be a collection of subsets of $M$ such that for all $x \in G_{n}$ and $0 \leq j<n$ we have that $g^{j}(x) \in G_{n-j}$. Let $B \subset M$ and let $x \in B$ be a point such that

$$
\#\left\{j \geq 1: x \in G_{j} \text { and } g^{j}(x) \in B\right\}=+\infty .
$$


Consider $\mathrm{O}^{+}(x)$ to be the positive orbit of $x$ and let $T: \mathcal{O}^{+} \cap B \rightarrow \mathcal{O}^{+} \cap B$ be the function defined by $T(y)=g^{\varphi(y)}(y)$, where $\varphi(y)=\min \left\{j \in \mathbb{N}: y \in G_{j}\right.$ and $g^{j}(y) \in$ $B\}$. Then, if

$$
\limsup _{n} \frac{1}{n} \#\left\{1 \leq j \leq n: x \in G_{j} \text { and } g^{j}(x) \in B\right\} \geq \theta>0,
$$

we have that

$$
\liminf _{n \rightarrow \infty} \frac{1}{n} \sum_{j=0}^{n-1} \varphi\left(T^{j}(x)\right) \leq \theta^{-1} .
$$

Using this lemma we are able to prove that

Lemma 3.8. The first hyperbolic time function of $g$ is integrable:

$$
\int n_{1}(x) d \mu<+\infty
$$

Proof. Observe that by Lemma 3.5. the set $H^{\theta}(c, \delta, g)$ has full measure. Since $\mu$ is ergodic, by Birkhoff's Ergodic Theorem, to show the integrability of $n_{1}$ is enough to verify that for every $x \in H^{\theta}(c, \delta, g)$ we have

$$
\lim _{n \rightarrow \infty} \frac{1}{n} \sum_{j=0}^{n-1} n_{1}\left(g^{n_{j}(x)}(x)\right) \leq \theta^{-1},
$$

where we set $n_{0}(x)=0$. Indeed, taking $G_{j}=H_{j}(c, \delta, g)$ and $B=H^{\theta}(c, \delta, g)$ as in Lemma 3.7 above, it follows from Remark 3.6 that if $x \in G_{n}$, then $g^{j}(x) \in G_{n-j}$ for every $0 \leq j<n$. Observe that by the definition, for every $x \in H^{\theta}(c, \delta, g)$ we have that

$$
\limsup _{n} \frac{1}{n} \#\left\{1 \leq j \leq n: x \in G_{j} \text { and } g^{j}(x) \in B\right\} \geq \theta>0 .
$$

At last, we have that $\varphi(x)=\min \left\{j \in \mathbb{N}: x \in H_{j}(c, \delta, g)\right.$ and $\left.g^{j}(x) \in H^{\theta}(c, \delta, g)\right\}=$ $n_{1}(x)$. Thus, the inequation above follows straightforwardly from Lemma 3.7.

An increasing sequence $\left(a_{k}\right)_{k \in \mathbb{N}}$ of natural numbers is called nonlacunary if

$$
\lim _{k \rightarrow \infty} \frac{a_{k+1}}{a_{k}}=1 .
$$

In 10, this notion is used in the context of equilibrium states to prove existence and uniqueness of a special type of weak Gibbs measure, called nonlacunary Gibbs measure. Therein, they proved that the integrability of the first hyperbolic time implies nonlacunarity. Here, we slightly generalize this result. Let $\gamma: \mathbb{R}^{+} \rightarrow \mathbb{R}^{+}$be a bijection. We say that an increasing sequence $\left(a_{k}\right)_{k \in \mathbb{N}}$ is $\gamma$-nonlacunary if

$$
\lim _{k \rightarrow \infty} \frac{a_{k+1}-a_{k}}{\gamma\left(a_{k}\right)}=0 .
$$

In particular, if $\gamma$ is the identity, a $\gamma$-nonlacunary sequence is just a nonlacunary sequence.

Lemma 3.9. If $\gamma^{-1}$ denotes the inverse function of $\gamma$, assume that for every $r>0$ the function $\gamma^{-1} \circ\left(r n_{1}\right)$ is $\mu$-integrable. Then, for $\mu$-a.e. $x \in M$ the sequence $n_{j}(\cdot)$ of its hyperbolic times is $\gamma$-nonlacunary. 
Remark 3.10. Before proving this lemma, we remark that the growth rate of $n_{i+1}-$ $n_{i}$ is obtained by analysing the decay of the measure of

$$
Q_{n}=H_{n} \backslash\left(\bigcup_{j<n} H_{j}\right)=\left\{x \in M: n_{1}(x)=n\right\} .
$$

If we know a priori that $\mu\left(Q_{n}\right)$ decays in a particular way, then we may take $\gamma(t)$ growing less than $t$ at infinity. For example, in [10, the authors show that every potential $\phi$ with low variation has a unique equilibrium state $\mu_{\phi}$. There, they used that $\mu_{\phi}$ is an expanding measure and $\mu\left(Q_{n}\right)$ decays exponentially. So, in this setting, the hypothesis of the previous lemma is easily satisfied for any function of the form $\gamma(t)=t^{p}$, where $p>0$.

Proof. Let $D$ be the set of points for which the sequence $n_{j}(\cdot)$ fails to be $\gamma$ nonlacunary. For each $r>0$, define $L_{r}(n)=\left\{x \in M: n_{1}(x) \geq r \gamma(n)\right\}$. If $x \in D$, then there exists a rational number $r>0$, and there are infinitely many values of $i$ such that $n_{i+1}(x)-n_{i}(x) \geq r \gamma\left(n_{i}(x)\right)$. By Remark 3.6. the latter implies that

$$
n_{1}\left(g^{n_{i}}(x)\right)=n_{i+1}(x)-n_{i}(x) \geq r \gamma\left(n_{i}(x)\right) .
$$

So, there are arbitrarily large values of $n$ such that $x \in g^{-n}\left(L_{r}(n)\right)$. In other words, $D$ is contained in the set

$$
L=\bigcup_{r \in \mathbb{Q} \cap(0,+\infty)} \bigcap_{k=0}^{\infty} \bigcup_{n \geq k} g^{-n}\left(L_{r}(n)\right) .
$$

Since $\mu$ is invariant, we have $\mu\left(g^{-n}\left(L_{r}(n)\right)\right)=\mu\left(L_{r}(n)\right)$ for all $n$. Then

$$
\begin{aligned}
\sum_{n=1}^{\infty} \mu\left(L_{r}(n)\right)=\sum_{n=1}^{\infty} \sum_{n_{1} \geq r \gamma(n)} \mu\left(Q_{n_{1}}\right) & =\sum_{m=1}^{\infty} \sum_{n=1}^{\left[\gamma^{-1}(m / r)\right]} \mu\left(Q_{m}\right) \\
& \leq \sum_{m=1}^{\infty} \gamma^{-1}(m / r) \mu\left(Q_{m}\right) .
\end{aligned}
$$

Thus, using the hypothesis that $\gamma^{-1}\left(n_{1}(\cdot) / r\right)$ is integrable,

$$
\sum_{n=1}^{\infty} \mu\left(L_{r}(n)\right) \leq \sum_{m=1}^{\infty} \gamma^{-1}(m / r) \mu\left(Q_{m}\right)=\int \gamma^{-1}\left(n_{1}(x) / r\right) d \mu(x)<\infty .
$$

By the Borel-Cantelli lemma, this implies that $L$ has measure zero. It follows that $\mu(D)=\mu(L)=0$, as claimed.

Corollary 3.11. If $\mu$ is an invariant expanding ergodic measure, then the sequence of hyperbolic times is nonlacunary.

Proof. Observe that $n_{1}$ is integrable by Lemma 3.8. To finish the proof, just put $\gamma(t)=t$ in Lemma 3.9

Lemma 3.12. For $\mu$-a.e. $x \in M$ we have that given $\epsilon>0$ small enough, if $B_{\epsilon}(n, x)$ is a dynamical ball at $x$ for $f$, then there exists a periodic point in $B_{n}(x, \epsilon)$, with period less than $n+K(n, x, \epsilon)$, where

$$
\limsup _{n \rightarrow \infty} \frac{K(n, x, \epsilon)}{n}=0 .
$$


Proof. Let us consider as before $g=f^{\ell}$ with $\ell$ chosen in such a way that $\mu$-almost all $x \in M$ have a nonlacunary infinite sequence $\left(n_{k}\right)_{k}$ of $g$-hyperbolic times (Lemma 3.5 and Corollary 3.11). We can assume that $x$ is such a point on $\operatorname{supp}(\mu)$, the support of $\mu$. Let $\epsilon>0$ and $n \geq 1$ be fixed with $\epsilon<\delta$ and let $k$ be such that $\ell n_{k}<n \leq \ell n_{k+1}$.

From the uniform continuity of $f$ it follows that there exists $\gamma=\gamma(\epsilon)<\epsilon$ such that for all $y \in M$ and $0 \leq k \leq \ell$,

$$
f^{k}(B(y, \gamma)) \subset B\left(f^{k}(y), \epsilon\right)
$$

As a consequence we have

$$
B_{g, n_{k+1}}(x, \gamma) \subset B_{f, n}(x, \epsilon) .
$$

Let $V(x)=g^{-n_{k+1}}\left(B\left(g^{n_{k+1}}(x), \gamma\right)\right)$ be the $g$-hyperbolic pre-ball around $x$ of length $n_{k+1}$. Since $n_{k+1}$ is a $g$-hyperbolic time for $x$ and $g^{-j}$ is a contraction on $B\left(g^{n_{k+1}}(x), \gamma\right)$ for all $1 \leq j \leq n_{k+1}$, it follows that $g^{n_{k+1}}(V(x))=B\left(g^{n_{k+1}}(x), \gamma\right)$ and that $V(x)=B_{g, n_{k+1}}(x, \gamma)$.

As $f$ is strongly transitive on the support of $\mu$, we can find $r>0$ and $N(\epsilon) \in \mathbb{N}$ such that for all $y \in \operatorname{supp}(\mu)$ we have $B(y, r) \subset f^{j}(B(x, \gamma))$ for some $j \leq N(\epsilon)$.

Without loss of generality, we may assume that $n$ satisfies $e^{-4 c n} \gamma<r$ which gives immediately $V(x)=B_{g, n_{k+1}}(x, \gamma) \subset B(x, r)$. Thus, we conclude that

$$
V(x) \subset f^{j}\left(B\left(f^{\ell n_{k+1}}(x), \gamma\right)\right)=f^{j}\left(f^{\ell n_{k+1}}(V(x))\right)
$$

for some $j \leq N$. Put $K(n, x, \epsilon)=\ell n_{k+1}+j-n$. By Brower's Fixed Point Theorem, we have that

$$
f^{n+K(n, x, \epsilon)}: V(x) \rightarrow f^{n+K(n, x, \epsilon)}(V(x))
$$

has a fixed point. Using the nonlacunarity of $\left(n_{k}\right)_{k}$ we get that

$$
\frac{K(n, x, \epsilon)}{n} \leq \frac{\ell n_{k+1}-n+N(\epsilon)}{n} \leq \frac{\ell n_{k+1}-\ell n_{k}+N(\epsilon)}{n_{k}} \rightarrow 0 \quad \text { when } k \rightarrow \infty .
$$

This finishes the proof of this lemma.

\section{Proof of Theorem $\mathrm{A}$}

In this section we prove Theorem $\mathrm{A}$. We need to prove that given an ergodic expanding measure $\mu, \epsilon>0, \eta>0$ small enough and $q$ an $\eta$-slowly varying function, for $\mu$-almost every point $x \in M$ we have that the nonuniform dynamical ball $\widetilde{B}_{n}(x, \epsilon, q)$ has a periodic point with period less than $n+K(n, x, \epsilon, \eta)$, where

$$
\lim _{\eta \rightarrow 0} \limsup _{n \rightarrow \infty} \frac{K(n, x, \epsilon, \eta)}{n}=0 .
$$

Proof of Theorem A, By Lemma 3.5 and Corollary 3.11, there exists $\ell$ such that almost every $x \in \operatorname{supp}(\mu)$ has infinitely many hyperbolic times $n_{1}<n_{2}<\ldots$ for $g=f^{\ell}$ and that $\left(n_{k}\right)$ is a nonlacunary sequence. Assume that $\eta<c$ and $\epsilon<\delta$. Denote by $\widetilde{B}_{n}(x, \epsilon, q)$ the nonuniform $(n, \epsilon, q)$-dynamical ball with respect to $f$ and by $\widetilde{B}_{n}^{\ell}(x, \epsilon, q)$ the $(n, \epsilon, q)$-dynamical ball with respect to $f^{\ell}$.

Observe that since $\|D f\|$ is uniformly bounded from above and, by the Mean Value inequality, given $\epsilon>0$ we may choose $\alpha=\alpha(\epsilon)<\epsilon$ such that given $y \in M$ and $0 \leq k \leq \ell$,

$$
f^{k}(B(y, r \alpha)) \subset B\left(f^{k}(y), r \alpha\right)
$$


for every $r>0$. As a consequence, given $m \in \mathbb{N}$, we have that

$$
\widetilde{B}_{\ell m}(x, \alpha(\epsilon), q) \subset \widetilde{B}_{m}^{\ell}(x, \epsilon, q) .
$$

Given $n \in \mathbb{N}$ big enough, take $\ell n_{i} \geq n>\ell n_{i-1}$ to be two consecutive hyperbolic times for $x$. By the definition of an $\eta$-slowly varying function, we have that $q\left(g^{n}(x)\right) \leq e^{\ell n \eta} q(x)$. Observing that $g_{x}^{-j}$ is a contraction for $1 \leq j \leq n_{i}$, we have that

$$
B\left(g^{n_{i}}(x), e^{-2 \ell n_{i} \eta} q(x)^{-2} \epsilon\right) \subset g^{n_{i}}\left(\widetilde{B}_{n_{i}}^{\ell}(x, \epsilon, q)\right) .
$$

Now, we choose $n_{i+s}$ a hyperbolic time for $x$ such that

$$
n_{i+s-1}<\frac{c+\eta}{c} n_{i} \quad \text { and } \quad n_{i+s} \geq \frac{c+\eta}{c} n_{i} .
$$

Observe that the choice of $n_{i+s}$ implies that $e^{-c\left(n_{i+s}-n_{i}\right)} \epsilon \leq e^{-n_{i} \eta} \epsilon$. Since $\left.B_{n_{i+s}-n_{i}}^{\ell}\left(g^{n_{i}}(x), \epsilon\right) \subset B\left(g^{n_{i}}(x), e^{-2 c\left(n_{i+s}-n_{i}\right)} q(x)^{-2} \epsilon\right) \subset B\left(g^{n_{i}}(x)\right), e^{-2 n_{i} \eta} q(x)^{-2} \epsilon\right)$, we have that

$$
g^{n_{i+s}-n_{i}}\left(B_{n_{i+s}-n_{i}}^{\ell}\left(g^{n_{i}}(x)\right)\right)=B\left(g^{n_{i+s}}(x), q(x)^{-2} \epsilon\right) \subset g^{n_{i+s}}\left(\widetilde{B}_{n_{i}}^{\ell}(x, \epsilon, q)\right) .
$$

Choosing $\alpha$ as in equation (7) and using that $\ell n_{i}>n$, we have that

$$
B\left(f^{\ell n_{i+s}}(x), q(x)^{-2} \epsilon\right) \subset f^{\ell n_{i+s}}\left(\widetilde{B}_{n}(x, \alpha, q)\right) .
$$

Since $f$ is strongly transitive on $\operatorname{supp}(\mu)$, we have that for $n$ big enough and some $0 \leq J \leq N\left(q(x)^{-2} \epsilon\right)$, the map $f^{\ell n_{i+s}+J}$ restricted to $\widetilde{B}_{n}(x, \alpha, q)$ is onto $\widetilde{B}_{n}(x, \alpha, q)$. Thus, by Brower's Fixed Point Theorem, $f$ has a periodic point on $\widetilde{B}_{n}(x, \alpha, q) \subset \widetilde{B}_{n}(x, \epsilon, q)$. Define

$$
K(n, x, \epsilon, \eta)=\ell n_{i+s}+J-n .
$$

Then $f$ has a periodic point with period at most $n+K(n, x, \epsilon, \eta)$. To finish the proof, we need to check that

$$
\lim _{\eta \rightarrow 0} \limsup _{n \rightarrow \infty} \frac{K(n, x, \epsilon, \eta)}{n}=0 .
$$

In fact, since $\left(n_{k}\right)$ is a nonlacunary sequence, we have that $n_{i} / n_{i-1}$ and $n_{i+s} /$ $n_{i+s-1}$ converge to 1 when $n$ goes to infinity (observe that $i$ goes to infinity when $n$ goes to infinity). By the left-hand side of equation (8) above:

$$
\frac{n_{i+s}}{n_{i}} \leq \frac{n_{i+s}}{n_{i+s-1}} \frac{c+\eta}{c}
$$

Observing that since $n_{i-1} \leq n \leq n_{i}$ and $n_{i} / n_{i-1} \rightarrow 1$ when $n$ goes to infinity, we have that $n_{i} / n \rightarrow 1$ when $n$ goes to infinity. Using this and the equation above, we conclude that

$$
\lim _{n \rightarrow \infty} \frac{n_{i+s}}{n} \leq \frac{c+\eta}{c}
$$

In particular,

$$
\limsup _{\eta \rightarrow 0} \limsup _{n \rightarrow \infty} \frac{K(n, x, \epsilon, \eta)}{n}=\lim _{\eta \rightarrow 0} \limsup _{n \rightarrow \infty} \frac{n_{i+s}+J-n}{n} \leq \lim _{\eta \rightarrow 0} \frac{c+\eta}{c}-1=0,
$$

since $0 \leq J \leq N\left(q(x)^{-2} \epsilon\right)$, and so Theorem $\mathrm{A}$ is proved. 


\section{ACKNOWLEDGEMENTS}

Part of this paper was written during a visit to IMPA and IBILCE/UNESP. The author gratefully acknowledges the hospitality of these institutions and the financial support of CNPq, CAPES, FAPERJ, FAPEAL and FAPESP. The author is also grateful to N. Muniz for several useful discussions and to F. Ledrappier, A. Katok, W. Sun and M. Viana for suggestions.

\section{REFERENCES}

[1] J. F. Alves and V. Araújo. Random perturbations of nonuniformly expanding maps. Astérisque, 286:25-62, 2003. MR 2052296(2005e:37058)

[2] J. F. Alves, C. Bonatti and M. Viana. SRB measures for partially hyperbolic systems whose central direction is mostly expanding. Invent. Math., 140:351-398, 2000. MR 1757000 (2001j:37063b)

[3] L. Arnold. Random dynamical systems. Springer-Verlag, 1998. MR.1723992 (2000m:37087)

[4] S. Vaienti, B. Saussol, and S. Troubetzkoy. Recurrence and Lyapunov exponents for diffeomorphisms. Moscow Mathematical Journal, 3:189-203, 2003. MR 1996808 (2004f:37016)

[5] R. Bowen. Periodic orbits for hyperbolic flows. Amer. J. Math., 94:1-30, 1972. MR0298700 $(45: 7749)$

[6] R. Bowen. Some systems with unique equilibrium states. Mathematical Systems Theory, 3:193-202, 1974. MR0399413 (53:3257)

[7] G. Liu, C. Liang and W. Sun. Approximation properties on invariant measure and Oseledec splitting in non-uniformly hyperbolic systems. Trans. Amer. Math. Soc., 361:1543-1579, 2009. MR2457408 (2009m:37085)

[8] A. Katok. Lyapunov exponents, entropy and periodic points of diffeomorphisms. Publ. Math. IHES, 51:137-173, 1980. MR573822 (81i:28022)

[9] K. Oliveira. Equilibrium states for certain non-uniformly hyperbolic systems. Ergod. Th. \& Dynam. Sys., 23:1891-1906, 2003. MR2032493 (2005b:37047)

[10] K. Oliveira and M. Viana. Thermodynamical formalism for robust classes of potentials and non-uniformly hyperbolic maps. Ergodic Theory and Dynamical Systems, 28(2):501-533, 2008. MR2408389 (2009b:37056)

[11] V. I. Oseledets. A multiplicative ergodic theorem: Lyapunov characteristic numbers for dynamical systems. Trans. Moscow Math. Soc., 19:197-231, 1968. MR0240280 (39:1629)

[12] Ya. B. Pesin. Characteristic Lyapunov exponents and smooth ergodic theory. Russian Math. Surveys, 32(4):55-114, 1977.

[13] V. Pinheiro. Expanding measures. Preprint, 2008, http://arxiv.org/abs/0811.2545. To appear in Ann. Inst. H. Poincaré Anal. Non Linéaire.

[14] V. Pliss. The location of separatrices of periodic saddle-point motion of systems of secondorder differential equations. Diff. Uravnenija, 7:906-927, 1971. MR0285770(44:2987)

[15] W. Sun and X. Tian. Pesin set, closing lemma and shadowing lemma in $C^{1}$ non-uniformly hyperbolic systems with limit domination. Preprint, 2010, arXiv:1004.0486.

[16] P. Varandas. Non-uniform specification and large deviations for weak Gibbs measures, arXiv:0906.3350v2, February 2010.

Instituto de Matemática, Universidade Federal de Alagoas, 57072-090 Maceió, AL, BrazIL

E-mail address: krerley@gmail.com 